ACADEMIA ROMÂNĂ
Rev. Roum. Chim.,
Revue Roumaine de Chimie
http://web.icf.ro/rrch/

\title{
TOPOLOGICAL PROPERTIES OF CHEMICAL STRUCTURES
}

\author{
Zheng-Qing CHU, ${ }^{\text {a }}$ Muhammad Kamran SIDDIQUI, ${ }^{\text {b,* }}$ \\ Muhammad Faisal NADEEM ${ }^{\mathrm{b}}$ and Muhammad NAEEM ${ }^{\mathrm{c}}$

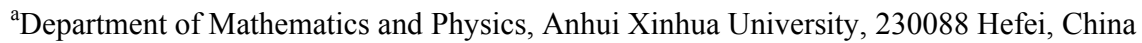 \\ ${ }^{b}$ Department of Mathematics, COMSATS University Islamabad, Lahore Campus, 54000, Pakistan \\ ${ }^{\mathrm{c}}$ Department of Mathematics and Statistics, Institute of Southern Punjab, Multan, 66000, Pakistan
}

Received May 5, 2018

Graph theory plays an important role in modeling and designing any chemical structure or network. The molecular topological descriptors are the numerical invariants of a molecular graph and are very useful for predicting their bioactivity. In this paper, we have studied the chemical graph of crystal structure of titanium difluoride $\mathrm{TiF}_{2}$, crystallographic structure of cuprite $\mathrm{Cu}_{2} \mathrm{O}$ and Boron-Carbon crystal structure of $\boldsymbol{P} \overline{\mathbf{4}} \boldsymbol{m} \mathbf{2} \boldsymbol{B} \boldsymbol{C}_{3}[\boldsymbol{s}, \boldsymbol{t}, \boldsymbol{n}]$. We have discussed and computed exact results for degree based topological indices namely Zagreb indices, Zagreb coindices and Sanskruti index.
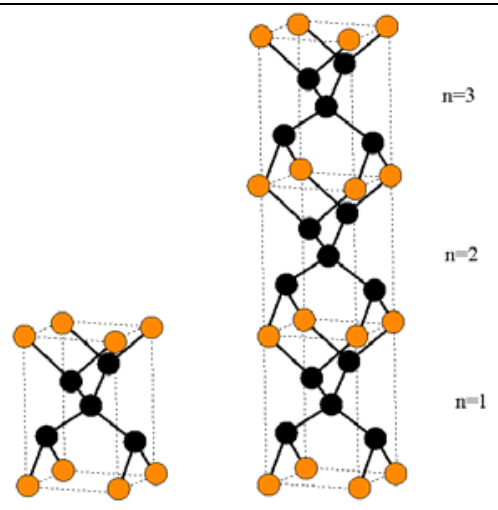

(c)

\section{INTRODUCTION}

Graph theory is a standout amongst the most extraordinary and one of a kind branch of mathematics by which the showing of any structure is made possible. As of late, it achieves much consideration among scientists on account of its extensive variety of utilizations in Computer science, electrical systems (network), interconnected systems (network), biological networks, and in chemistry, and so forth. The chemical graph theory (CGT) is the quickly developing zone among scientists. It helps in comprehension about the basic properties of a molecular graph. There are a considerable measure of molecular compounds, which have assortment of utilizations in the fields of business, commercial, industrial, pharmaceutical chemistry, in every day life and in research facility.
A relationship exists between molecular compounds and their atomic structures. The control and examination of molecular compounds data is made possible utilizing molecular descriptors. Chemical graph theory is a branch of mathematical chemistry in which apparatuses of graph theory are applied to model the chemical phenomenon mathematically. Likewise, it identifies with the nontrivial uses of graph theory for solving molecular problems. This theory contributes a noticeable part in the field of chemical sciences.

A new subject namely Chem-informatics which is a combination of chemistry, mathematics and information science helps to analyzes $(Q S A R)$ and $(Q S P R)$ connections that are used to predict the bioactivity and physiochemical properties of chemical compounds. A topological index is a

* Corresponding author: kamransiddiqui75@gmail.com 
numerical esteem that is processed mathematically from the molecular graph. It is related with chemical constitution demonstrating for correlation of chemical structure with numerous physical, chemical properties and biological activities, see details. ${ }^{1,10}$

For a given $G=(V, E)$ graph where $V$ to be the vertex set and $\boldsymbol{E}$ to be the edge set of $\boldsymbol{G}$. The degree $a_{p}$ of $p$ is the quantity of edges of $G$ incident with $p$. The length of a most limited path in a graph $\boldsymbol{G}$ is a distance $d(p, q)$ amongst $p$ and $q$ represented by a polynomial, a numerical value or by matrix form. There are sure sorts of topological indices primarily eccentric based, degree based and distance based indices etc. In this paper, we have dealt with degree based topological indices.

The Zagreb indices were conceived in 1972 by Gutman and Trinajestic, ${ }^{11,12}$ they are characterized as:

$$
\begin{aligned}
& M_{1}(G)=\Sigma_{p q \in E(G)}\left(d_{p}+d_{c}\right) \\
& M_{2}(G)=\Sigma_{p q \in E(G)}\left(d_{p} \times a_{c}\right)
\end{aligned}
$$

In 2008, Došlić put forward the first Zagreb coindex and second Zagreb coindex, defined as ${ }^{4}$

$$
\begin{aligned}
& \overline{M_{1}}=\overline{M_{1}}(G)=\Sigma_{p q \notin E(G)}\left[a_{p}+d_{q}\right] \\
& \overline{M_{2}}=\overline{M_{2}}(G)=\Sigma_{p q \notin E(G)} d_{p} \times d_{q}
\end{aligned}
$$

In 2016, I. Gutman et al. ${ }^{7}$ proves the following Theorems:

Let $G$ be a graph with $|F(G)|$ vertices and $|B(G)|$ edges. Then

$\overline{M_{1}}(G)=2|E(G)|(\mid V(G)-1)-M_{1}(G)$.

Let $G$ be a graph with $|\gamma(G)|$ vertices and $\left|E^{\prime}(G)\right|$ edges. Then

$$
\overline{M_{2}}(G)=2|E(G)|^{2}-\frac{1}{2} M_{1}(G)-M_{2}(G)(6)
$$

In 2016, S. M. Hosamani ${ }^{14}$ introduced the Sanskruti index $\boldsymbol{S}(\boldsymbol{G})$ for a molecular graph $\boldsymbol{G}$, defined as:

$$
S(G)=\sum_{n q F R(G)}\left(\frac{s_{p} \times S_{q}}{S_{p}+S_{q}-2}\right)^{3}
$$

For further study and research about the topological indices see. ${ }^{1,6,13,16,21-28}$

\section{Main results}

In this section, additive topological indices mainly first and second zegreb indices, first and second zegreb coindices and Sanskruti index of $C u_{2} O[m, n, t], \quad T i F_{2}[m, n, t] \quad$ and $P \overline{4} m 2 B C_{\mathrm{g}}[s, t, n]$ are computed. Moreover, the exact values are derived for these indices of the chemical graphs $C u_{2} O\left[m_{1}, t\right], \quad T i \Gamma_{2}[m, n, t]$ and $\bar{\Gamma} \overline{4} m 2 S C_{3}\lceil s, t, n\rceil$.

\section{Crystallographic structure of $\mathrm{Cu}_{2} \mathrm{O}$}

Among different transition metal oxides, $\mathrm{Cu}_{2} \mathrm{O}$ has pulled in extensive consideration as of late attributable to its recognized properties and nontoxic nature, minimal effort, plenitude, and basic creation process. These days, the promising uses of $\mathrm{Cu}_{2} \mathrm{O}$ chiefly concentrate on chemical sensors, solar oriented cells, photocatalysis, lithium-particle batteries and catalysis. The chemical graph of Crystallographic structure of $\mathrm{Cu}_{2} \mathrm{O}$ portrayed in Figure 1 and Figure 2, for more data about this structure see. ${ }^{2,30,31}$ Let $G \cong C u_{2} \boldsymbol{O}[\boldsymbol{m}, \boldsymbol{n}, \boldsymbol{t}]$ be the chemical graph of $\mathrm{C}^{\prime} u_{2} \mathrm{O}$ with $m \times n$ unit cells in the plane and $t$ layers. We develop this graph first by taking $m \times n$ units in the $\boldsymbol{m} \boldsymbol{m}$-plane and after that leveling up in $t$ layers. The quantity of vertices and edges of $C u_{2} \sigma[m, n, t]$ are $(m+1)(n+1)(t+1)+5 m m t$ and $8 m m t$, respectively.

In $C u_{2} O[m, n, t]$ the number zero degree vertices is 4 , the number of one degree vertices is $4 m+4 n+4 t-0$, the number of two degree vertices is $4 m n t+2 m n+2 m t+2 n t-4 n-$. $-4 m-4 t+6$ and the number of four degree vertices is $2 n m t-n m-n t-m t+n+m+1$.

In the next Theorem, we have computed the exact result of first and second Zagreb index for the chemical graph $\mathrm{Cu}_{2} \mathrm{O}[m, n, t]$.

Theorem 3.1. Consider the graph of $G \cong \mathbf{C} u_{2} O[m, n, t]$ with $m, n, t \geq 1$, then its first and second Zagreb index is equal to,

$$
\begin{gathered}
M_{1}(G)-48 m n t-8 m n-8 m t-8 n t+4 m+4 n+1 t \\
M_{2}(G)=64 m m-16 m n-16 m t-26 n t+8 m+8 n+8 t
\end{gathered}
$$




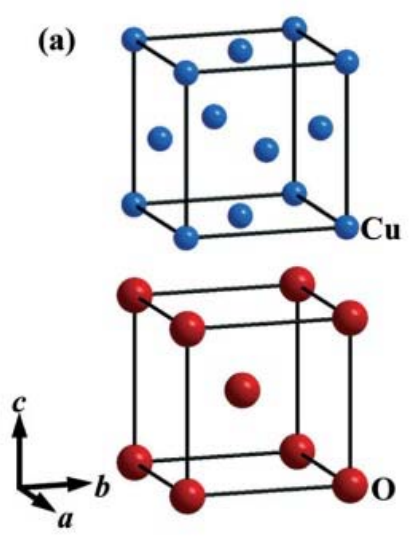

(b)

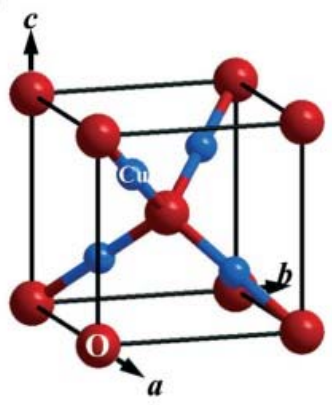

Fig. 1 - Crystallographic structure of $\mathrm{Cu}_{2} \mathrm{O}$. (a) In lattice of $\mathrm{Cu}_{2} \mathrm{O}$ the structural characteristics of the atoms of $\mathrm{Cu}$ and $\mathrm{O}$. The lattice of $\mathrm{Cu}_{2} \mathrm{O}$ is formed by interpenetrating the lattices of $\mathrm{Cu}$ and $\mathrm{O}$ into each other. (b) Unit cell of $\mathrm{Cu}_{2} \mathrm{O}$, where copper and oxygen atoms are shown in small blue and in large red spheres. In the lattice of $\mathrm{Cu}_{2} \mathrm{O}$, every $\mathrm{Cu}$ atom is connected with two $\mathrm{O}$ atoms, and every $\mathrm{O}$ atom is connected with four $\mathrm{Cu}$ atoms.

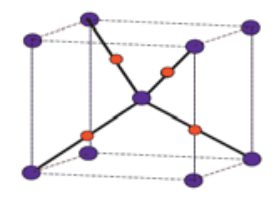

(a)

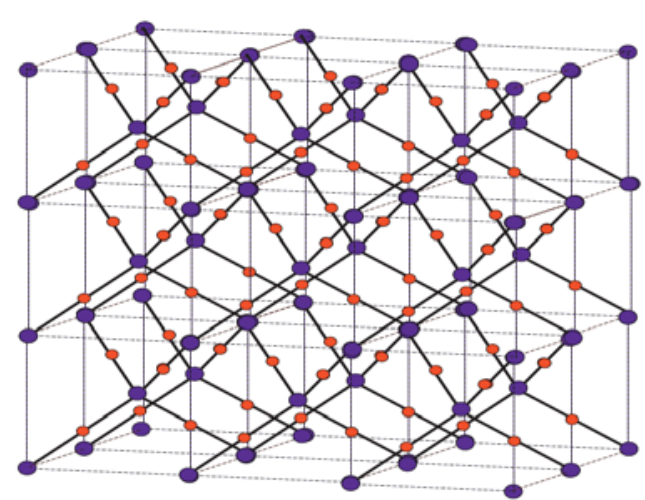

(b)

Fig. 2 - Crystallographic structure of $\mathrm{Cu}_{2} \mathrm{O}[3,2,3]$.

Table 1

Degree based partition of edges of $\mathrm{Cu}_{2} \mathrm{O}[\mathrm{m}, n, t]$, of end vertices of each edge

\begin{tabular}{c|c}
\hline$\left(\mathbf{d}_{\mathbf{p}}, \mathbf{d}_{\mathbf{q}}\right)$ & Frequency \\
\hline$(1,2)$ & $4 n+4 m+4 t-8$ \\
$(2,2)$ & $4 n m+4 n t+4 m t-8 n-8 m-8 t+12$ \\
$(2,4)$ & $4(2 n m t-n m-n t-m t+n+m+t-1)$ \\
\hline
\end{tabular}

Proof. Let $\boldsymbol{G}$ be the crystallographic structure of computed as below: $\boldsymbol{C} \boldsymbol{u}_{2} \boldsymbol{O}[\boldsymbol{m}, \boldsymbol{n}, \boldsymbol{t}]$. The first Zagreb index is

$$
\begin{gathered}
M_{1}(G)=\sum_{p q \in E(G)}\left(d_{p}+d_{q}\right) \\
M_{\perp}(G)=(4 m+4 m+4 t-8)(1+2)+(4 m m+4 m t+4 n t-8 m-8 n-8 t+12)(2+2) \\
+(8 m m t-4 m n-4 m t-4 n t+4 m+4 n+4 t-4)(2+4) \\
M_{1}(G)=48 m n t-8 m n-8 m t-8 n t+4 m+4 n+4 t .
\end{gathered}
$$

By using Table 1 and Equation (1) the second Zagreb index are computed as below:

$$
\left.M_{2}, G\right)=\Sigma_{p G \in \vec{s}(G)}\left(d_{p} d_{q}\right)
$$




$$
\begin{aligned}
M_{2}(G)=(4 m & +4 n+4 t-8) \cdot(1 \times 2)+(4 m n+4 m t+4 n t-8 m-8 n-8 t+12)(2 \times 2) \\
& +(8 m n t-4 m n-4 m t-4 n t+4 m+4 n+4 t-4)(2 \times 4) \\
& =64 m n t-16 m n-16 m t-16 n t+8 m+8 n+8 t
\end{aligned}
$$

The first and second Zagreb coindices for the chemical graph $\mathrm{Cu}_{2} O[\mathrm{~m}, \boldsymbol{n}, \mathrm{t}]$ computed are in the next Theorem.
Theorem 3.2. Consider the graph of $G \cong C u_{2} O[m, n, t]$ with $m, n, t \geq 1$, then its first and second Zagreb coindices are equal to,

$$
\begin{aligned}
& \overline{M_{1}}(G)=80 m^{2} n^{2} t^{2}+16 m n t(m+1)(n+1)(t+1)-64 m n t+8 m+8 m t \\
& +8 n t-4 m-4 n-4 t \\
& \bar{M}(G)=128 m^{2} n^{2} t^{2} \quad 88 m n t \quad \text { । } 20 m n \text { । } 20 m t \text { । } 20 n t \quad 10 m \quad 10 n \quad 10 t
\end{aligned}
$$

Proof. Let $G$ be the crystallographic structure of $C u_{2} O[m, n, t]$. Then by using Equations (3),(5) and Theorem 1 first Zagreb coindex is computed as below:

$$
\begin{aligned}
& M_{1}(G)=\sum_{p G(G)}\left(a_{p}+a_{q}\right) \\
& \left.\overline{M_{1}}(G)=2|E(G)| G|V(G)|-1\right)-M_{1}(G) \\
& =2(8 m n t)((m+1)(n+1)(t+1)+5 m n t-1) \\
& -(48 m n-8 m n-8 m t-8 n t+4 m+4 n+4 t) \\
& =80 m^{2} n^{2} t^{2}+16 m n t(m+1)(n+1)(t+1)-64 m n t+8 m n+8 m t \\
& +8 n t-4 m-4 n-4 t .
\end{aligned}
$$

Now, by using Equations (4),(6) and Theorem 1 second Zagreb coindex is computed as below:

$$
\begin{aligned}
& \overline{M_{2}}(G)=\Sigma_{p G E E(G)}\left(d_{p} d_{q}\right) \\
& =2|E(G)|^{2}-\frac{1}{2} M_{1}(G)-M_{2}(G) \\
& \overline{M_{2}}(G)=2(8 m n t)^{2}-\frac{1}{2}(48 m n t-8 m n-8 m t-8 n t+4 m+4 n+4 t) \\
& -(64 m m t-16 m-16 m t-16 n t+8 m+8 n+8 t) \\
& =128 m^{2} n^{2} t^{2}-88 m n t+20 m n+20 m t+20 n t-10 m-10 n-10 t
\end{aligned}
$$

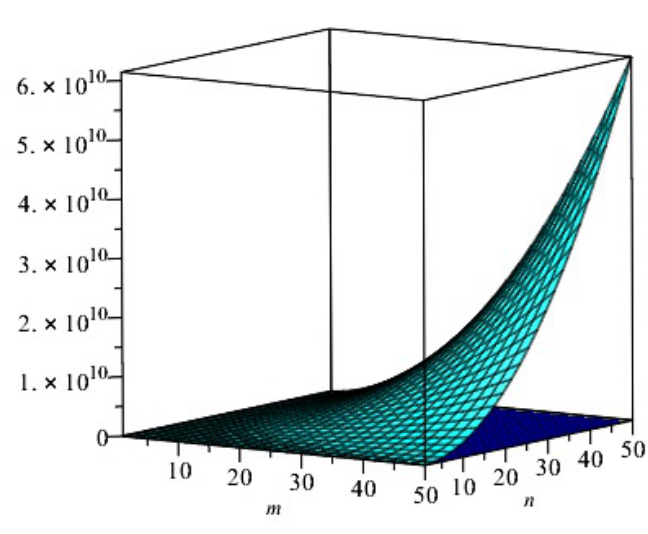

(a)

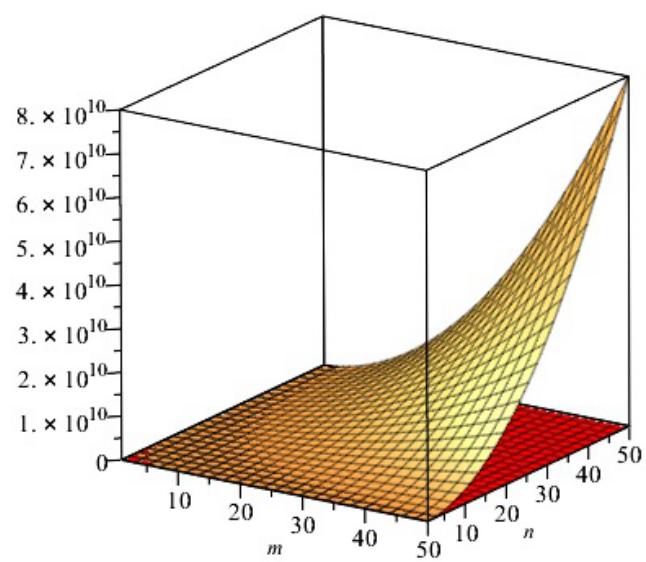

(b)

Fig. 3 - Comparison of indices of $G \cong C u_{2} O[m, n, t]$, for fix $t=10$ : in (a) first Zagreb index $M_{1}(G)$ and first Zagreb coindex $\bar{M}_{1}(G)$ are compared. The blue and cyan colors represents $M_{1}(G)$ and $\bar{M}_{1}(G)$, respectively. Its easy to see that in the given domain $\bar{M}_{1}(G)$ is more dominating. In (b) second Zagreb index $M_{2}(G)$ and second Zagreb coindex $\bar{M}_{2}(G)$ are compared. The red and gold colors represents $M_{2}(G)$ and $\bar{M}_{2}(G)$, respectively. Its easy to see that in the given domain $\bar{M}_{2}(G)$ is more dominating. over the values of $\bar{M}_{2}(G)$ are largest and the values of $M_{1}(G)$ are smallest. 
Table 2

Based on sum of degrees of neighborhood of end vertices of each edge the partition of edges of $\mathrm{Cu}_{2} \mathrm{O}[\mathrm{m}, n, t]$, with $m, n, t \geq 2$

\begin{tabular}{l|l}
\hline \multicolumn{1}{c|}{$\left(\mathbf{S}_{\mathbf{p}}, \mathbf{S}_{\mathbf{q}}\right)$} & \multicolumn{1}{c}{ Frequency } \\
\hline$(2,4)$ & $4 m+4 n+4 t-8$ \\
$(4,6)$ & $4 m n+4 m t+4 n t-8 m-8 n-8 t+12$ \\
$(5,8)$ & $4 n+4 m+4 t-8$ \\
$(6,8)$ & $4 m n+4 m t+4 n t-8 m-8 n-8 t+12$ \\
$(8,8)$ & $8 m n t-8 m n-8 n t+8 m+8 n+8 t-8$ \\
\hline
\end{tabular}

The Table 2 shows partition of edges of the chemical graph $\mathbf{C u _ { 2 }} O[m, n, t]$ depending on the sum of degrees of the neighbouring vertices of end vertices of each edge. The next Theorem shows the exact value of Sanskruti index of $C u_{2} O\left[m_{1} n, t\right]$.
Theorem 3.3. Consider the graph $G \cong C u_{2} \sigma[m, n, t]$ with $m, n, t \geq 2$, then its Sanskruti index $S(G)$ is equal to

$$
\begin{aligned}
& S(G)=\frac{262144 m n t}{343}-\frac{137292 \mathrm{~mm}}{343}-\frac{137292 \mathrm{mt} t}{343}-\frac{137292 \mathrm{nt}}{343}+\frac{118974696 \mathrm{n}}{456533}+\frac{118974696 t}{456533} \\
& +\frac{118974696 m}{456533}-\frac{557 \cdot 374 n}{456533}
\end{aligned}
$$

Proof. Let $\boldsymbol{G}$ be the crystallographic structure of $C u_{2} \boldsymbol{O}[\boldsymbol{m}, \boldsymbol{r}, \boldsymbol{t}]$. Then by using Table 2 and equation (7) the Sanskruti index $\boldsymbol{S}(\boldsymbol{G})$ is computed as follows.

$$
\begin{gathered}
S(G)=\sum_{p q \in E(G)}\left(\frac{S_{p} \times S_{q}}{S_{p}+S_{q}-2}\right)^{3} \\
S(G)=(4 m+4 n+4 t-8)\left(\frac{2 \times 4}{2+4-2}\right)^{3}+(4 m n+4 n t+4 m t-8 m-8 n-8 t \\
+12)\left(\frac{4 \times 6}{4+6-2}\right)^{3}+(4 m+4 n+4 t-8)\left(\frac{5 \times 8}{5+8-2}\right)^{3} \\
+(4 m n+4 n t+4 m t-8 m-8 n-8 t+12)\left(\frac{6 \times 8}{6+8-2}\right)^{3}+(8 m n t-8 m n-8 m t-8 n t+8 m+ \\
8 n+8 t-8)\left(\frac{8 \times 8}{8+8-2}\right)^{3} \\
S(G)=\frac{262144 m n t}{343}-\frac{137292 m n}{343}-\frac{137292 m t}{343}-\frac{137292 n t}{343}+\frac{118974696 n}{456533} \\
+\frac{118974696 t}{456533}+\frac{118974696 m}{456533}-\frac{55213740}{456533}
\end{gathered}
$$

\section{Crystal Structure of Titanium Difluoride}

Titanium Difluoride is a water insoluble Titanium source for use in oxygen-sensitive applications, for example, metal production. Fluoride compounds have various applications in current advances and science, from oil refining and drawing to engineered organic chemistry and the making of pharmaceuticals.

The chemical graph of crystal structure of titanium difluoride $\operatorname{TiF}_{2}[\boldsymbol{m}, \boldsymbol{n}, \mathbf{t}]$ is described in Figure 4, for more details see., ${ }^{3,10}$ Let
$G \cong T_{l} F_{2}[m, n, t]$ be the chemical graph of $T i F_{2}$ with $\boldsymbol{m} \times \boldsymbol{n}$ unit cells in the plane and $\boldsymbol{t}$ layers. We construct this graph first by taking $m \times n$ unites in the $\boldsymbol{m} \boldsymbol{n}$-plane and then storing it up in $\boldsymbol{t}$ layers. The number of vertices and edges of $T \dot{i} F_{2}[m, n, t] \quad$ are $12 m n t+2 m n+2 m t+2 n t+m+n+t+1$ and $32 m m$, respectively.

In $T i F_{2}[M, n, t]$ the number of one degree vertices is 8 , the number of two degree vertices is $4 m+4 n+4 t-12$, the number of four degree 
vertices is $8 m n \dot{t}+4 m n+4 m t+4 n t-4 n-\quad$ vertices is $4 m n t-2(m n+m t+n t)+m$.

$-4 m-4 t+6$ and the number of eight degree

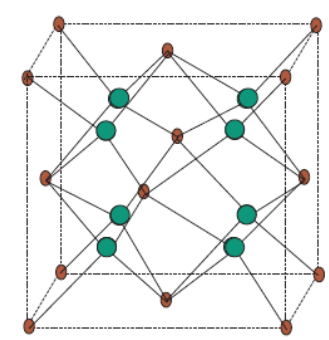

(a)

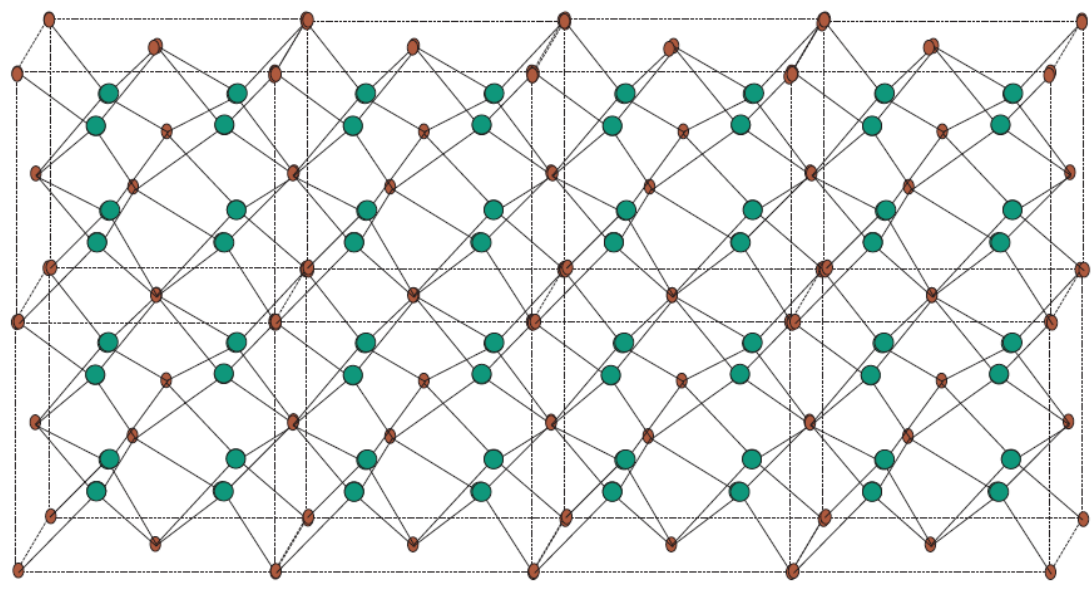

(b)

Fig. 4 - Crystal Structure Titanium Difluoride $T i F_{2}[m, n, t]$, (a) represents unit cell of of $T i F_{2}[m, n, t]$, with Tiatoms in red and $F$ atoms in green (b) crystal structure of $\mathrm{TiF}_{2}[4,1,2]$.

Table 3

Degree based partition of edges of $\mathrm{TiF}_{2}[\mathrm{~m}, n, t]$, of end vertices of each edge

\begin{tabular}{c|l}
\hline$\left(\boldsymbol{d}_{\boldsymbol{p}}, \boldsymbol{d}_{\boldsymbol{q}}\right)$ & Frequency \\
\hline$(1,4)$ & 8 \\
$(2,4)$ & $8(m+n+t-3)$ \\
$(4,4)$ & $16(m n+m t+n t)-16(m+n+t)+24$ \\
$(4,8)$ & $32 m n t-16(m t+m n+n t)-8$ \\
\hline
\end{tabular}

The exact result of the first and second Zagreb index for the chemical graph $T \dot{t} F_{2}[\boldsymbol{m}, \boldsymbol{n}, \boldsymbol{t}]$ is computed in the following theorem.
Theorem 4.1. Consider the graph $G \cong T_{1} F_{2}[m, n, t]$ with $m, n, t \geq 1$, then its first and second Zagreb index is equal to,

$$
\begin{aligned}
& M_{1}(G)=0[40 m n t-0(m n+m t+n t)+2(m+n+t)-1] \\
& M_{2}(G)=32[32 m n t-8(m n+m t+n t)+2(m+n+t)-1]
\end{aligned}
$$

Proof. Let $G \cong T F_{2}[m, n, t]$ be the crystal structure of titanium difluoride. Then by using

Table 3 and the equation (1) the first Zagreb index is computed as below:

$$
\begin{gathered}
M_{1}(G)=\sum_{p q E S(G)}\left(d_{p}+d_{q}\right) \\
M_{1}(G)=(8)(1+4)+(8 m+8 n+8 t-24)(2+4)+(16(m n+m t+n t)-16(m+n+ \\
t)+24)(8)+(32 m n t-16(m n+m t+n t)+8(m+n+t)-8)(4+8) \\
M_{1}(G)=384 m n t-64 m n-64 m t-64 n t+16 m+16 n+16 t-8
\end{gathered}
$$

Now, by using Table 3 and the equation (2) the second Zagreb index is computed as below:

$$
M_{2}(G)=\Sigma_{p q \in \bar{B}(G)}\left(d_{p} d_{q}\right)
$$

$M_{2}(G)=(8)(1 \times 4)+(8 m+8 n+8 t-24)(2 \times 4)+(16(m n+m t+n t)-16(m+n+$ $t)+24)(4 \times 4)+(32 m n t-16(m n+m t+n t)+8(m+n+t)-8)(4 \times 8)$ 


$$
M_{2}(G)=1024 m n t-256 m n-256 m t-256 n t+64 m+64 n+64 t-32
$$

The first and second Zagreb coindices for the chemical graph $T \dot{i} F_{2}[m, n, t]$ computed are in the next Theorem.
Theorem 4.2. Consider the graph of $G \cong T_{1} F_{2}[m, n, t]$ with $\boldsymbol{m}, \boldsymbol{n}, t>1$, then its first and second Zagreb coindices are equal to,

$$
\begin{aligned}
& \overline{M_{1}}(G)=64 m n t(12 m n t+2 m n+2 m t+2 n t+m+n+t) \\
& -381 m n t+61 m n+61 m t+64 n t-16 m-16 n-16 t+8 \text {. } \\
& \overline{M,}(G)=2048 m^{2} n^{2} t^{2}-1216 m n t+288 m n+288 m t+288 n t-72 m-72 n-72 t+36
\end{aligned}
$$

Proof. Let $\boldsymbol{G}$ be the crystallographic structure of $T i F_{\mathfrak{z}}[m, n, t]$. Then by using Equations (3),(5) and Theorem 3 first Zagreb coindex is computed as below:

$$
\begin{aligned}
& \overline{M_{1}}(G)=\sum_{p q E(G)}\left(d_{p}+d_{q}\right) \\
& M_{1}(G)=2|E(G)|(|V(G)|-1)-M_{1}(G) \\
& =2(32 m n t)(12 m n t+2 m m+2 n t+2 n t+m+n+t+1-1) \\
& -(384 m n t-64 m n-64 m t-64 n t+16 m+16 n+16 t-8) \\
& =64 m n t(12 m n t+2 m n+2 m t+2 n t+m+n+t) \\
& -384 m n t+64 m n+64 m t+64 n t-16 m-16 n-16 t+8,
\end{aligned}
$$

Now, by using Equations (4),(6) and Theorem 3 second Zagreb coindex is computed as below:

$$
\begin{gathered}
\overline{M_{2}}(G)=\Sigma_{p q E E(G)}\left(d_{p} d_{q}\right) \\
\overline{M_{2}}(G)=2|E(G)|-\frac{1}{2} M_{1}(G)-M_{2}(G) \\
=2(32 m n t)^{2}-\frac{1}{2}(384 m n t-64 m n-64 m t-64 n t+16 m+16 n+16 t-8) \\
-(1024 m n t-256 m n-256 m t-256 n t+64 m+64 n+64 t-32) \\
=2048 m^{2} n^{2} t^{2}-1216 m n t+288 m n+288 m t+288 n t-72 m-72 n-72 t+36
\end{gathered}
$$

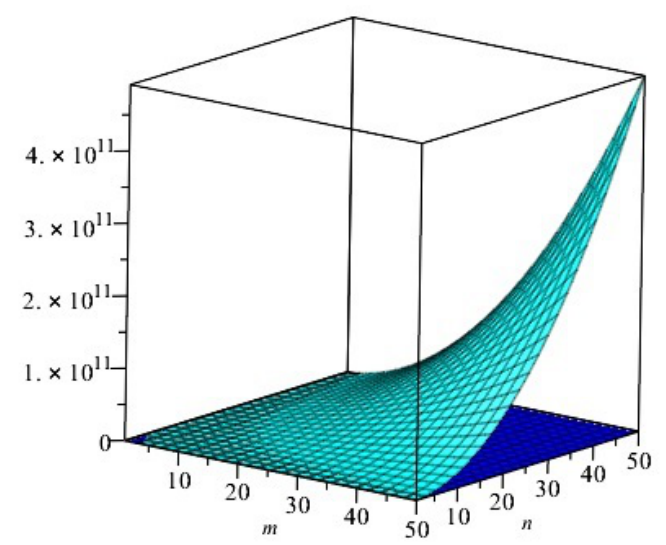

(a)

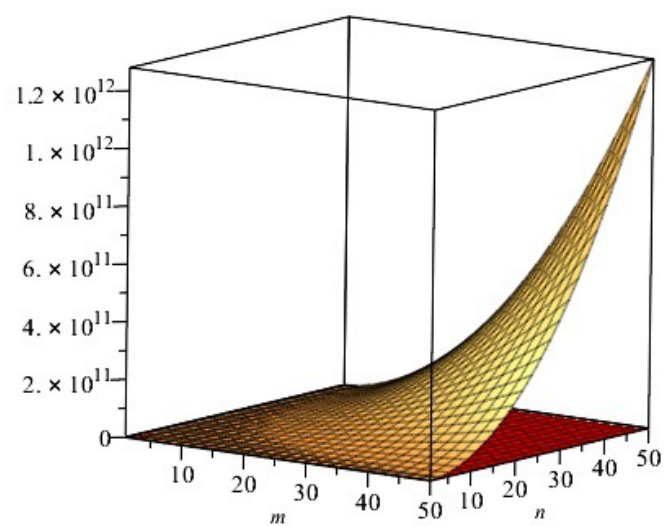

(b)

Fig. 5 - Comparison of indices of $G \cong \operatorname{TiF}_{2}[m, n, t]$ for fix $t=10$ : in (a) first Zagreb index $M_{1}(G)$ and first Zagreb coindex $\bar{M}_{1}(G)$ are compared. The blue and cyan colors represents $M_{1}(G)$ and $\bar{M}_{1}(G)$, respectively. Its easy to see that in the given domain $\bar{M}_{1}(G)$ is more dominating. In (b) second Zagreb index $M_{2}(G)$ and second Zagreb coindex $\bar{M}_{2}(G)$ are compared. The red and gold colors represents $M_{2}(G)$ and $\bar{M}_{2}(G)$, respectively. Its easy to see that in the given domain $\bar{M}_{2}(G)$ is more dominating. over the values of $\bar{M}_{2}(G)$ are largest and the values of $M_{1}(G)$ are smallest. 
Table 4

Edge partition of $\operatorname{TiF}_{2}[m, n, t], m, n, s \geq 2$ based on degree sum of end vertices of each edge

\begin{tabular}{l|l}
\hline \multicolumn{1}{c}{$\left(\mathbf{S}_{\mathbf{p}}, \mathbf{S}_{\mathbf{q}} \mathbf{)}\right.$} & Frequency \\
\hline$(4,13)$ & 8 \\
$(8,18)$ & $8(m+n+t-3)$ \\
$(13,16)$ & 16 \\
$(16,18)$ & $16(m n+m t+n t)-16(m+n+t)+8$ \\
$(16,24)$ & $32 m n t-16(m n+m t+n t)+8$ \\
$(18,32)$ & $8(m+n+t-2)$ \\
\hline
\end{tabular}

The Table 4 shows the edge partition of the chemical graph $T \dot{t} F_{2}[m, n, t], m, n, s \geq 2$ based on the degree sum of end vertices of each edge. The next Theorem shows the exact value of Sanskruti index of $T i F_{2}[m, n, t], m, n, s>2$.
Theorem 4.3. Consider the graph $G \cong T_{1} F_{2}[m, n, t]$ with $m, n, t \geq 2$, then its Sanskruti index $S(G)$ is equal to

$$
\begin{aligned}
& S(G)=\frac{276497416}{6859} m n t-\frac{99747937}{6859} m n+\frac{46760544}{6859} n t-\frac{1197467 n \mathrm{n}}{6859} m t+3888 m+3888 n+ \\
& 3888 t-\frac{197259525709696}{16875712125}
\end{aligned}
$$

Proof. Let $G$ be the crystallographic structure of $T \dot{i} F_{2}[\boldsymbol{m}, \boldsymbol{n}, \boldsymbol{t}]$. Then by using Table 4 and equation (7) the Sanskruti index $\boldsymbol{S}(\boldsymbol{G})$ is computed as follows.

$$
\begin{aligned}
& S(G)=\Sigma_{p_{7} \in E(G)}\left(\frac{s_{p} \times S_{q}}{s_{p}+S_{p}-2}\right)^{3} \\
& S(G)=(8)\left(\frac{4 \times 13}{4+13-2}\right)^{3}+8(m+n+t-3)\left(\frac{8 \times 18}{8+18-2}\right)^{3} \\
& +(16)\left(\frac{19 \times 16}{19+16-2}\right)^{3}+(16(m n+m t+n t)-16(m+n+t)+8)\left(\frac{16 \times 18}{16+18-2}\right)^{3} \\
& +(32 m m-16(m n+m t+n t)+8)\left(\frac{16 \times 74}{16+24-2}\right)^{3}+8(m+n+t-2)\left(\frac{18 \times 37}{18+32-2}\right)^{3} \\
& =\frac{226492416}{6859} m n t-\frac{39242892}{6859} m n+\frac{46760544}{6859} n t-\frac{113246208}{6859} m t+3888 m+3888 n \\
& +3888 t-\frac{187258525708696}{16375712125}
\end{aligned}
$$

Boron-Carbon crystal structure of $\overline{P 4} \mathrm{~m}_{2 B C}[s, t, n]$

The vast majority of Boron and Carbon systems denoted as B-C binary systems show high imperviousness to oxidation furthermore, response with ferrous metals, contrasted and the carbonbased materials. ${ }^{9,15,18}$ Boron carbide $B_{4} C$ is a hard crystal that can be created at surrounding pressure, 8,17 while $B$-doped diamond demonstrates a superconducting progress temperature of $4 \boldsymbol{K} .^{5}$ It is of extraordinary enthusiasm for diamond-like $B C_{x}$ systems to seek after predominant superhard crystals that are not just thermally and chemically more stable(steady) than diamond, yet in addition have intriguing electrical properties. ${ }^{9,20,29}$

Liu et al. ${ }^{19}$ investigated the crystal structures with particle swarm optimization (PSO) algorithm consolidated with first-principles structural optimizations. Three metallic setups, to be specific Pmma - $a, P_{m m a}-\mathrm{b}$ and $\bar{P} \overline{4} \boldsymbol{m} 2$ stages were revealed. With the bond resistance demonstrate, the Vickers hardness for all three stages is bigger than $60 \mathrm{GPa}$, demonstrating the superhard nature of these polymorphs, which ought to be tested experimentally. Furthermore, all stages have a superconductive transition at low temperature.

Let $G=P \overline{4} m Z B C_{\sharp}\lceil s, t, n]$ be the chemical graph of $P \overline{4} m 2 B C_{\mathrm{g}}$ with $s \times t$ unit cells in the plane and $\boldsymbol{n}$ layers. We construct this graph first by taking $s \times t$ unites in the $s t-$ plane and then storing it up in $n$ layers. Some description of the construction are given in Figure 6 and Figure 7. The number of vertices and edges of $P \overline{4} m 2 B C_{\mathrm{a}}[s, t, n] \quad$ are $\quad(s+1)(t+1)+$ 
$+n(2 s+1)(2 t+1)$ and $n(8 s t+2 s+2 t)$. In $P \overline{4} m Z B C_{g}[s, t, n]$ the number of one degree vertices is $2(s+t+2)$, the number of two degree vertices is $2(s t+2 n-3)$, the number of three degree vertices is $2 n(s+t)+2(n-1)$ $(s+t-2)$ and the number of four degree vertices is $4 s n t-2 s n-s t-2 t n+n+s+t$. The edges of $P \overline{4}^{4} m 2 B C_{3}|s, t, n|$ are partitioned into seven sets, say $E_{1}, E_{2}, E_{3}, E_{4}, E_{5}, E_{6}$ and $E_{7}$. The set $E_{1}$ contains $\delta$ edges $p q$, where $d_{p}=3$ and $a_{a}=1$. The set $E_{z}$ contains $2(s+t)-4$ edges $p q$, where $\bar{a}_{v}=4$ and $d_{a}=1$. The set $E_{3}$ contains $4(t+s+2 n-4)$ edges $p q$, where $a_{p}=3$ and $d_{a}=2$. The set $E_{4}$ contains $4(s-1)(t-1)$ edges $p q$, where $d_{v}=4$ and $x_{q}=2$. The set $E_{5}$ contains $4(n-1)(t+s-2)$ edges $p q$, where $a_{p}-i_{q}-3$. The set $E_{6}$ contains $2 n(s+t)+2(n-1)(s+t-2)$ edges $p q$, where $a_{v}=4$ and $\bar{a}_{v}=3$. The set $B_{7}$ contains $8 s n t-6 s n-4 s t-6 n t+4 s+4 n+4 t-4$ edges $p q$, where $d_{p}=a_{q}=4$. The edge partition is shown in Table 5 of $\bar{P} \overline{4} \boldsymbol{m} \boldsymbol{B} B C_{\mathfrak{n}}\lceil\boldsymbol{s}, \mathbf{t}, \boldsymbol{n}\rceil$ with $s, t, n \geq 1$.

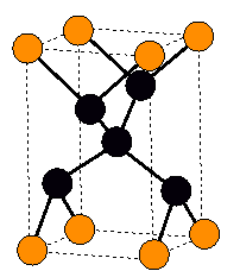

(b)

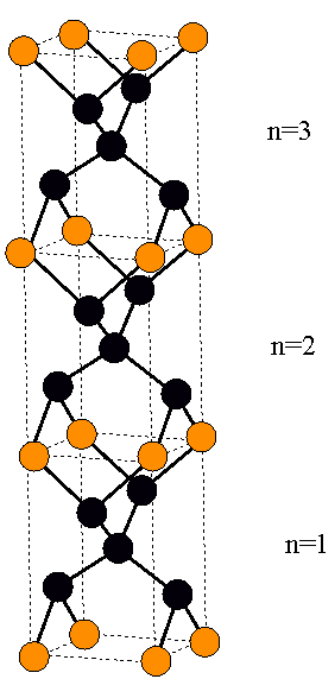

(c)

Fig. 6 - Crystal structures of $\boldsymbol{P} \overline{\mathbf{4}} \boldsymbol{m} \mathbf{2} \boldsymbol{B} C_{3}[s, t, n]$, (a) chemical graph of unit cell,

(b) $\boldsymbol{P} \overline{\mathbf{4}} \boldsymbol{m} 2 B C_{3}[1,1,3]$ with $s=t=1$ and $n=3$ layers. Carbon atom are black Boron atoms are brown.

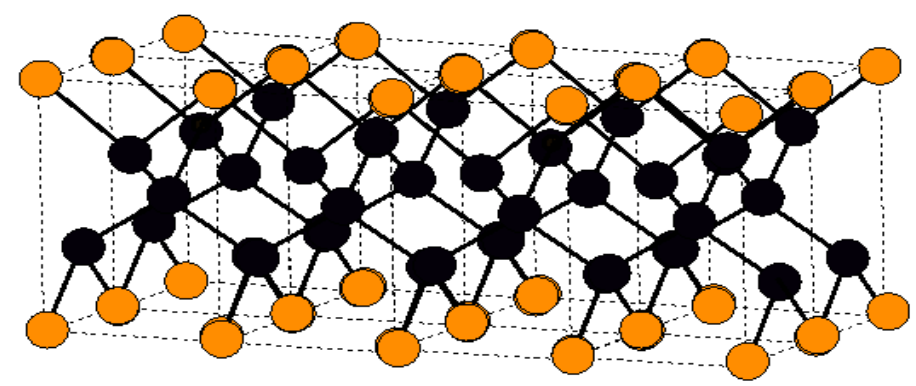

Fig. 7 - Crystal structures of $\bar{P} \overline{\mathbf{4}} \boldsymbol{m} \mathbf{2 B} C_{3}[\mathbf{4}, \mathbf{2}, 1]$.

Table 5

Degree based partition of edges of $\overline{\boldsymbol{P}} \overline{\mathbf{4}} \boldsymbol{m} \mathbf{2} \boldsymbol{B} C_{3}[s, t, n]$, of end vertices of each edge

\begin{tabular}{c|l}
\hline$\left(\boldsymbol{d}_{\boldsymbol{p}}, \boldsymbol{d}_{\boldsymbol{q}}\right)$ & Frequency \\
\hline$(3,1)$ & 8 \\
$(4,1)$ & $2(s+t)-4$ \\
$(3,2)$ & $4(t+s+2 n-4)$ \\
\hline
\end{tabular}


Table 5 (continued)

\begin{tabular}{l|l}
\hline$(4,2)$ & $4(s-1)(t-1)$ \\
$(3,3)$ & $4(n-1)(t+s-2)$ \\
$(4,3)$ & $2 n(s+t)+2(n-1)(s+t-2)$ \\
$(4,4)$ & $8 s n t-6 s n-4 s t-6 n t+4 s+4 n+4 t-4$ \\
\hline
\end{tabular}

In the following Theorems we have computed the first and second Zagreb indices, the first and second Zagreb coindices of the chemical graph $P \overline{4} m 2 B C_{3}[s, t, n]$.

$$
\begin{aligned}
& M_{1}(G)=64 s n t-10 s n-8 s t-10 n t-4 n \\
& M_{2}(G)=120 s n t-36 s n-32 s t-36 n t+4 s-6 n+4 t
\end{aligned}
$$

Proof. Let $\boldsymbol{G}$ be the graph of indices are computed as below:

$P 4 m 2 B C_{g}[s, t, n\rceil$. Then by using Table 5 and the equations (1),(2) the first and second Zagreb

$$
\begin{gathered}
M_{1}(G)=\sum_{p q \in E(G)}\left(d_{p}+\dot{a}_{q}\right) \\
M_{1}(G)=(8)(3+1)+(2 s+2 t-4)(4+1)+4(t+s+2 n-4)(3+2)+4(s-1)(t- \\
1)(4+2) \quad+4(n-1)(t+s-2)(3+3)+2(n-1)(t+s-2)(4+3) \\
+(8 s n t-6 s n-4 s t-6 n t+4 s+4 n+4 t-4)(4+4) \\
M_{1}(G)=64 s n t-10 s n-8 s t-10 n t-4 n \\
M_{2}(G)=\sum_{p q E R(G)}\left(a_{p} d_{q}\right)=H_{1}(G) \\
M_{2}(G)=(8)(3 \times 1)+(2 s+2 t-4)(4 \times 1)+4(t+s+2 n-4)(3 \times 2) \\
+4(s-1)(t-1)(4 \times 2)+4(n-1)(t+s-2)(3 \times 3)+2(n-1)(t+s \\
-2)(4 \times 3)+(8 s n t-6 s n-1 s t-6 n t+1 s+4 n+4 t-4)(4 \times 4) \\
M_{2}(G)=128 s n t-36 s n-32 s t-36 n t+4 s-8 n+4 t
\end{gathered}
$$

Theorem 5.2. Consider the graph of first and second Zagreb coindices are equal to, $G \cong P \overline{4} r 2 B C_{\mathbf{3}}[s, i, r\rceil$ with $m, n, t \geq$, then its

$$
\begin{aligned}
& \overline{M_{1}}(G)=2 n(8 s t+2 s+2 t)((s+1)(t+1)+n(2 s+1)(2 t+1)-1)-64 s n t-10 s n+ \\
& 10 n t+10 s t+4 n
\end{aligned}
$$

$\overline{M_{7}}(G)-2046 m^{2} n^{2} L^{\prime}-1216 m u+266 m n+266 m L+266 n t-72 m-72 n-72 t+36$

Proof. Let $\boldsymbol{G}$ be the crystallographic structure of computed as below:

$\boldsymbol{P} \overline{4} \boldsymbol{m} 2 \boldsymbol{B} \boldsymbol{C}_{\boldsymbol{g}}[\boldsymbol{s}, \boldsymbol{t} \boldsymbol{n}]$. Then by using Equations

(3),(5) and Theorem 5 first Zagreb coindex is

$$
\begin{aligned}
& \overline{M_{1}}(G)=\sum_{p q E(G)}\left(d_{p}+a_{q}\right) \\
& M_{1}(G)=2|E(G)|(|V(G)|-1)-M_{1}(G) \\
& =2(n(8 s t+2 s+2 t))((s+1)(t+1)+n(2 s+1)(2 t+1)-1) \\
& -(64 s n t-10 s n-8 s t-10 n t-4 n) \\
& =2 n(8 s t+2 s+2 t)((s+1)(t+1)+n(2 s+1)(2 t+1)-1)-64 s n t-10 s n+10 n t+ \\
& 10 s t+4 n .
\end{aligned}
$$

Now, by using Equations (4),(6) and Theorem 5 second Zagreb coindex is computed as below: 


$$
\begin{aligned}
& \overline{M_{2}}(G)=\Sigma_{p q E E(G)}\left(d_{p} d_{q}\right) \\
& \overline{M_{2}}(G)=2|E(G)|-\frac{1}{2} M_{1}(G)-M_{2}(G) \\
& =2(n(8 s t+2 s+2 t))^{2}-\frac{1}{2}(64 s n t-10 s n-8 s t-10 n t-4 n) \\
& -(128 s n t-36 s n-32 s t-36 n t+4 s-8 n+4 t) \\
& =2 n^{2}(8 s t+2 s+2 t)^{2}-160 s n t+31 s n+41 n t+37 s t+10 n-4 s-4 t
\end{aligned}
$$

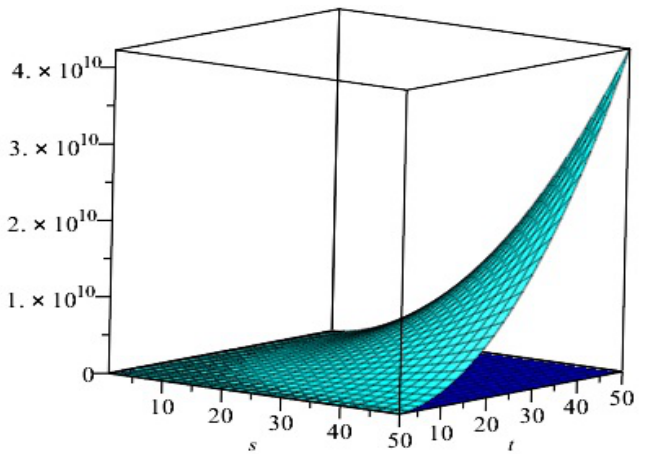

(a)

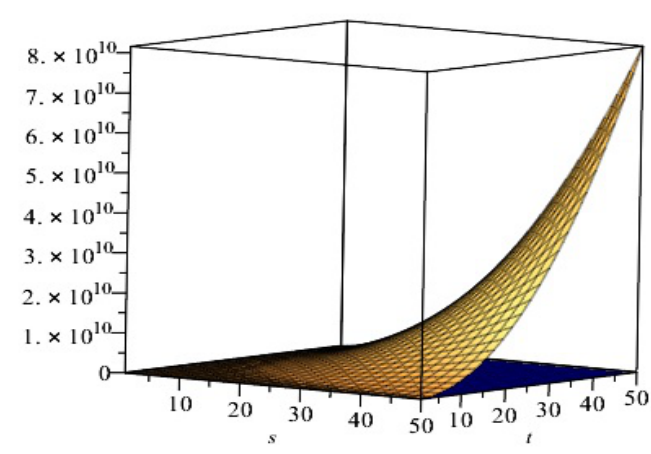

(b)

Fig. 8 - Comparison of indices of $G \cong P \overline{4} m 2 B C_{3}[s, t, n]$ for fix $t=10$ : in (a) first Zagreb index $M_{1}(G)$ and first Zagreb coindex

$\bar{M}_{1}(G)$ are compared. The blue and cyan colors represents $M_{1}(G)$ and $\bar{M}_{1}(G)$, respectively. Its easy to see that in the given domain $\bar{M}_{1}(G)$ is more dominating. In (b) second Zagreb index $M_{2}(G)$ and second Zagreb coindex $\bar{M}_{2}(G)$ are compared. The red and gold colors represents $M_{2}(G)$ and $\bar{M}_{2}(G)$, respectively. Its easy to see that in the given domain $\bar{M}_{2}(G)$ is more dominating. over the values of $\bar{M}_{2}(G)$ are largest and the values of $M_{1}(G)$ are smallest.

The Table 6 shows the edge partition of the chemical graph $P \overline{4} m 2 B C_{\mathrm{n}}[s, t, n], s, t, n \geq 2$ based on the degree sum of end vertices of each edge. The next Theorem shows the exact value of
Sanskruti index of $\bar{P} \overline{4} m 2 B C_{\mathfrak{n}}[s, t, n]$, $s, t, n \geq 2$.

Table 6

Edge partition of $P \overline{4} m 2 B C_{3}[s, t, n]$ based on degree sum of end vertices of each edge

\begin{tabular}{l|l}
\hline \multicolumn{1}{c|}{$\left(\mathbf{S}_{\mathbf{p}}, \mathbf{S}_{\mathbf{q}}\right)$} & Frequency \\
\hline$(7,3)$ & 8 \\
$(11,4)$ & $2(s+t-4)$ \\
$(7,6)$ & 8 \\
$(8,6)$ & $4(s+t)-16$ \\
$(9,6)$ & $8(n-1)$ \\
$(12,8)$ & $4(s-1)(t-1)$ \\
$(10,9)$ & $8(n-1)$ \\
$(10,10)$ & $4(n-1)(t+s-1)$ \\
$(12,7)$ & $8 n$ \\
$(14,8)$ & $2 n(s+t-4)$ \\
$(15,10)$ & $2(n-1)(s+t-2)$ \\
$(14,11)$ & 8 \\
$(15,11)$ & $4(s+t-4)$ \\
$(15,12)$ & $2(s+t-4)$ \\
$(16,12)$ & $4(t-2)(s-2)$ \\
$(14,15)$ & $8(n-1)$ \\
$(15,15)$ & $4(n-1)(s+t-4)$ \\
$(15,16)$ & $4(n-1)(s+t-4)$ \\
$(16,16)$ & $8 s n t-14 s n-8 s t-14 n t+14 s+28 n+14 t-28$ \\
\hline
\end{tabular}


Theorem 5.3. Consider the graph Sanskruti index $S(G)$ is equal to $G \cong T i F_{2}[m, n, t]$ with $m, n, t \geq 2$, then its

$$
\begin{aligned}
& S(G)=\frac{29496215665267292}{989590583625} n+\frac{10515259529957}{949104000} t+\frac{10515259529557}{949104000} s+\frac{49904}{125} n(s+t-4) \\
& -\frac{29360128}{3375} s m-\frac{29360126}{3375} n t+\frac{16777216}{3375} s n t-\frac{16777216}{3375} s t+\frac{4096(4 s-4)(t-1)}{27} \\
& +\frac{490669538420125(4 n-4)(s+t-4)}{(390297362112}+\frac{3375000(2 n-2)(s+t-2)}{12167}+\frac{884736(4 t-8)(s-2)}{(2197} \\
& -\frac{67996570672683862395978593}{2097952710974303008300}
\end{aligned}
$$

Proof. Let $\boldsymbol{G}$ be the crystallographic structure of $T_{\dot{u}} F_{2}[\boldsymbol{m}, \boldsymbol{n}, \boldsymbol{t}]$. Then by using Table 4 and

equation (7) the Sanskruti index $\boldsymbol{S}(\boldsymbol{G})$ is computed as follows.

$$
\begin{aligned}
& S(G)=\sum_{p q \in E(G)}\left(\frac{S_{p} \times S_{q}}{S_{p}+S_{p}-2}\right)^{3} \\
& S(G)=(8)\left(\frac{7 \times 3}{7+9-2}\right)^{3}+2(s+ \pm-4)\left(\frac{11 \times 4}{11+4-2}\right)^{3}+(8)\left(\frac{7 \times 6}{7+6-2}\right)^{3} \\
& +(1(s+t)-16)\left(\frac{8 \times 6}{8+6-2}\right)^{3}+8(n-1)\left(\frac{9 \times 6}{9+6-2}\right)^{3}+1(s-1)(t-1)\left(\frac{12 \times 8}{12+8-2}\right)^{3} \\
& +8(n-1)\left(\frac{10 \times 9}{10+9-2}\right)^{3}+4(n-1)(t+s-4)\left(\frac{10 \times 10}{10+10-2}\right)^{3}+8 n\left(\frac{12 \times 7}{12+7-2}\right)^{3} \\
& +2 n(s+t-4)\left(\frac{14 \times 8}{14+8-2}\right)^{3}+2(n-1)(s+t-2)\left(\frac{15 \times 10}{15+10-2}\right)^{3}+8\left(\frac{14 \times 11}{14+11-2}\right)^{3} \\
& +4(s+t-4)\left(\frac{15 \times 11}{15+11-2}\right)^{3}+2(s+t-4)\left(\frac{15 \times 12}{15+12-2}\right)^{3}+4(t-2)(s-2)\left(\frac{16 \times 12}{16+12-2}\right)^{3} \\
& +8(n-1)\left(\frac{14 \times 15}{14+15-2}\right)^{3}+4(n-1)(s+t-4)\left(\frac{15 \times 15}{15+15-2}\right)^{3} \\
& +4(n-1)(s+t-4)\left(\frac{15 \times 16}{15+16-2}\right)^{3} \\
& +(8 m t-14 m-8 s t-14 n t+14 s+28 n+14 t-28)\left(\frac{16 \times 16}{16+16-2}\right)^{3} \\
& =\frac{23496215663287232}{963590563625} n+\frac{10515259523957}{949+04000} t+\frac{10515259523957}{949104000} s+\frac{43904}{125} n(s+t-4) \\
& -\frac{29360128}{3375} s n-\frac{29360128}{3375} n t+\frac{16777216}{3975} s n t-\frac{16777216}{3975} s t+\frac{4096(4 s-4)(t-1)}{27} \\
& +\frac{490669538420125(4 n-4)(s+t-4)}{390297362112}+\frac{3375000(2 n-2)(s+t-2)}{12167}+\frac{884736(4 t-8)(s-2)}{2197} \\
& -\frac{67996570672683862335978593}{2033852310824309008300}
\end{aligned}
$$

\section{CONCLUSIONS}

In this paper, we have studied and computed some degree based topological indices for the chemical graph of the crystal structure of titanium difluoride $T \dot{t} F_{2}$, crystallographic structure of cuprite $\mathrm{Cu}_{2} \mathrm{O}$ and Boron-Carbon crystal structure of $\bar{P} \overline{4} m Z B C_{\eta}[s, t, n]$. The exact results have been computed of the first and second Zagreb indices, first and second Zagreb coindices and Sanskruti index for $T i F_{2}, C u_{2} O$ and $\bar{P} \overline{4} m Z B C_{3}\lceil s, t, n\rceil$.

The graphical representations of Zagreb indices of $C u_{2} O[m, n, t]$ and $T \dot{L} F_{2}\left[m, n, t_{-}^{-}\right.$are depicted in Figure 3 and Figure 5 for certain values of $(m, n)$ and fix $t$. By varying the value of $m, n$ the Zagreb indices behaves differently. The graphical representations of Zagreb indices of $P \overline{4} m 2 B C_{\mathfrak{g}}[s, t, n]$ are depicted in Figure 5 for certain values of $s, t$ and fix $\boldsymbol{n}$. By varying the value of $s, t$ the Zagreb indices behaves differently.

Acknowledgements. The authors would like to thanks the two anonymous reviewers for their very constructive comments that helped us to enhance the quality of this manuscript. The authors would like to express their sincere gratitude to the Natural Science Foundation for the Higher Education Institutions of Anhui Province of China (no. KJ2019A0875 and no. KJ2019A0876). 


\section{REFERENCES}

1. A. Q. Baig, M. Imran, W. Khalid and M. Naeem, Canadian J. Chem., 2017, 95, 674-686.

2. K. Chen, C. Sun, S. Song and D. Xue, Cryst. Eng. Comm., 2014, 16, 52-57.

3. F. A. Cotton, G. Wilkinson, C. A. Murillo and M. Bochmann, "Advanced Inorganic Chemistry", John Wiley and Sons, 1999.

4. T. Došlić, Ars Math. Contemp, 2008, 1, 66-80.

5. E. A. Ekimov, V. A.Sidorov, E. D. Bauer, N. N. Mel'nik, N. J. Curro and J. D. Thompson, Nature, 2004, 428, 5425-5437.

6. W. Gao, M. K. Siddiqui, M. Imran, M. K. Jamil and M. R. Farahani, Saudi Pharma. J., 2016, 24, 258-264.

7. I. Gutman, B. Furtula, Ž. K. Vukićević and G. Popivoda, MATCH Commun. Math. Comput. Chem., 2015, 74, 5-16.

8. X. J. Guo, J. L. He, Z. Y. Liu, Y. J. Tian, J. Sun and H.T. Wang, Phys Rev B, 2006, 73, 104-115.

9. X. J. Guo, J. L. He, B. Xu, Z. Y. Liu, D. L. Yu and Y. J. Tian, J. Phys. Chem. C, 2007, 111, 13679-13683.

10. N. N. Greenwood and A. Earnshaw in "Chemistry of the Elements", second edition, Butterworth, UK, 1997.

11. I. Gutman and K. C. Das, MATCH Commun. Math. Comput. Chem., 2004, 50, 83-92.

12. I. Gutman and N. Trinajstć, Chem. Phys. Lett., 1972, 17, 535-538.

13. X. Li and I. Gutman, Mathematical aspects of Randić type molecular structure descriptors, mathematical chemistry monographs No. 1, Kragujevac, 2006.

14. S. M. Hosamani, J. Appl. Math. Comput., 2016, 6, 19-29.

15. Q. K. Hu, Q. H. Wu, Y. M. Ma, L. J. Zhang, Z. Y. Liu and J. L. He, Phys. Rev. B, 2006, 73, 214-116.
16. Y. Hu, X. Li, Y. Shi, T. Xu and I. Gutman, MATCH Commun. Math. Comput. Chem., 2005, 54, 425-434.

17. U. Jansson and J. O. Carlsson, Thin Solid Films, 1985, 124,101-107.

18. I. Kurakevych, J. Superhard Mater., 2009, 31, 139-157.

19. H. Y. Liu, Q. A. Li , L. Zhu and Y. M. Ma, Phys Lett A, 2011, 375, 771-774.

20. Z. Y. Liu, J. L. He, J. Yang, X. J. Guo, H. Sun and H. T. Wang, Phys. Rev. B, 2006, 73, 17-21..

21. M. K. Siddiqui, M. Imran, A. Ahmad, Appl. Math. Comput., 2016, 280, 132-139.

22. M. K. Siddiqui, M. Naeem, N. A. Rahman and M. Imran, JOAM, 2016, 18, 884-892.

23. N. Soleimani, M. J. Nikmehr and H. A. Tavallaee, $J$. Natn. Sci. Foundation, Sri Lanka, 2015, 43, 127- 133.

24. D. Vukičević and B. Furtula, J. Math. Chem., 2009, 46, $1369-1376$.

25. B. Basavanagoud, W. Gao, S. Patil, V. R. Desai, K. G. Mirajkar and B. Pooja, Appl. Mathem. Nonlinear Scie., 2017, 2, 285-298.

26. D. Liu, C. Wang and S. Wang, Appl. Mathem. Nonlinear Scie., 2018, 3, 419-426.

27. J. A. Aledo, L. G. Diaz, S. Martinez and J. C. Valverde, Appl. Mathem. Nonlinear Scie., 2018, 3, 593-602.

28. M. Knor, R. Skrekovski and A. Tepeh, Appl. Mathem. Nonlinear Scie., 2018, 3, 433-446.

29. J. Yang, H. Sun, J. L. He, Y. Tian and C. F. J. Chen, J. Phys. Condens. Matter., 2007, 19, 34-43

30. B. D. Yuhas and P. Yang, J. Am. Chem. Soc., 2009, 131, 3756-3461.

31. J. Zhang, J. Liu, Q. Peng, X. Wang and Y. Li, Chem. Mater., 2006, 18, 867-871. 
